\title{
Study of Tracer Diffusion Mechanism in Amorphous Metal
}

\author{
P. H. Kien, ${ }^{1}$ H. V. Hue, ${ }^{2}$ and P. K. Hung ${ }^{3}$ \\ ${ }^{1}$ Department of Physics, Thai Nguyen University of Education, Luong Ngoc Quyen, Thai Nguyen, Vietnam \\ ${ }^{2}$ Faculty of General Science, Ho Chi Minh City University of Food Industry, 140 Le Trong Tan, Ho Chi Minh, Vietnam \\ ${ }^{3}$ Department of Computational Physics, Ha Noi University of Science and Technology, 1 Dai Co Viet, Ha Noi, Vietnam
}

Correspondence should be addressed to P. H. Kien, huukienpham@yahoo.com

Received 11 March 2012; Revised 27 May 2012; Accepted 12 June 2012

Academic Editor: E. Mittemeijer

Copyright ( $) 2012$ P. H. Kien et al. This is an open access article distributed under the Creative Commons Attribution License, which permits unrestricted use, distribution, and reproduction in any medium, provided the original work is properly cited.

The statistical relaxation (SR) simulation has been conducted to study the behavior of simplexes and bubbles (BB) in amorphous Co metal containing $2 \times 10^{5}$ atoms. The simulation reveals that the fraction of 4 -simplex increases and $n$-simplex $(n>4)$ decreases depending upon relaxation degree. The simulation found that a large number of BB vary upon relaxation degree, which could play a role of diffusion vehicle for Co atoms in amorphous matrix. The idea of the diffusion mechanism in amorphous metal is described as follows: the elemental atomic movement includes a jump of neighboring atom into the BB and then a collective displacement of a large number of atoms around $\mathrm{BB}$.

\section{Introduction}

The diffusion behavior in amorphous metals (AMs) has been intensively studied by both experiment and simulation for long times [1-8]. It is found that there are many specific properties of diffusion in AM compared to crystal matters. For example, the tracer diffusivity in a well-relaxed sample is much slower than the one in an as-quenched sample $[9,10]$. This relaxation effect is interpreted by the reduction of vacancies in supersaturation until the relaxation is over. In the well-relaxed sample, conversely, the tracer atoms diffuse via collective movement of a group of neighboring atoms. The experimental studies in [1113] on isotope effect, pressure dependence, and irradiationenhanced diffusivity are sometimes in contradiction with the diffusion described above. Furthermore, there is not a clear definition of vacancy. Computer simulation, on the other hand, reveals unstableness of vacancies in amorphous matrix. Several works found a continuous spectrum of spherical voids in amorphous matter, but their size is less than atomic radius [14-16]. The free volume and two-level state theories are also employed to interpret the diffusion behavior of amorphous matter, but they cannot properly describe the diffusivity in some amorphous matters such as $\mathrm{Ti}_{60} \mathrm{~N}_{40}$ and $\mathrm{Fe}_{40} \mathrm{Ni}_{40} \mathrm{~B}_{20}$ which show the cooperative activated movement more like diffusivity in solid state than in liquid [2, 17-20]. In [21], Sietsma and Thijsse analyzed different types of holes in amorphous matters and found that the number of holes surrounded by ten or more atoms decreases strongly in a well-relaxed sample. Furthermore, they argue the importance of big holes for atomic diffusivity.

Our previous study shows that amorphous matters suffer from a number of spherical voids, the size of which is closed to atomic size. Because the concentration of those voids weakly depends on temperature, it is very sensitive to the relaxation degree. We call them "native vacancy," which fully disappears until the amorphous matters transform into crystalline solid. However, the analysis of this study is based only on the geometrical consideration. Therefore, the account of potential barrier could provide more accurate elucidation of the voids for diffusion in amorphous matters. The aim of the present work is the study of simplexes and their role of them in diffusion in Co amorphous metals by using SR simulation method as a way to clarify diffusion mechanism in amorphous matters.

\section{Calculation Method}

The simulation has been conducted for the sample consisting of $2 \times 10^{5}$ atoms in a cubic box with periodic boundary conditions. We use the Pak-Doyama potential [16], and 


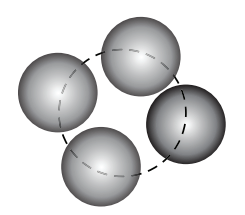

(a)

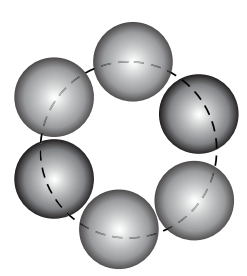

(b)

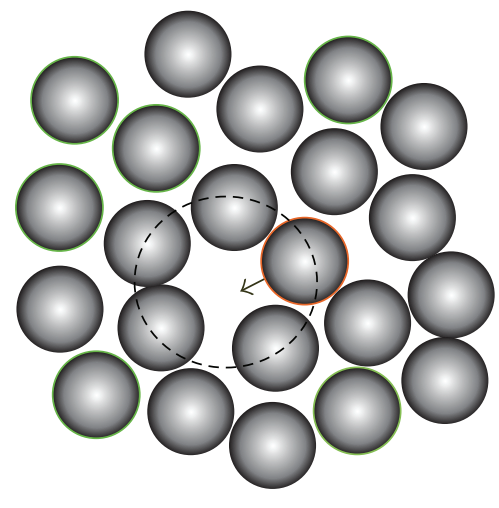

(c)

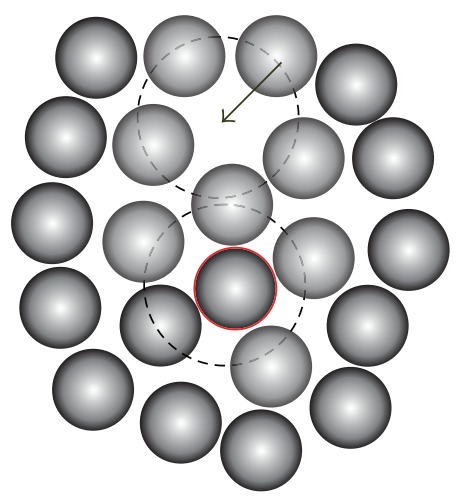

(d)

Figure 1: The schematic illustration of simplexes: (a) 4-simplex; (b) 6-simplex; (c) 5-simplex and neighboring atoms; (d) the new 5-simplex and neighboring atoms after RDA. The dash circle presents the CSP of four atoms, and the arrow is a path along which the MA jumps into CSP.

the density is taken from real amorphous metal. The interatomic potential has the following form:

$$
\begin{aligned}
\varphi\left(r_{i j}\right)= & -0.12812\left(r_{i j}-1.82709\right)^{4}+1.15421\left(r_{i j}-2.50849\right)^{2} \\
& -0.13448, \quad 0 \leq r_{i j} \leq 3.44 \AA
\end{aligned}
$$

where $r_{i j}$ is the distance between $i$ and $j$ atoms. Molecular dynamic (MD) simulation could reproduce the structure of AM; however, this method is time consuming. Thus, we employ the SR method, which provides the structure of AM similar to that of the low-temperature MD model. Accordingly, each atom moves by a length $d r$ in the direction of the force acting on it from all remaining atoms. The length $d r$ is equal to $0.01-0.4 \AA$. This movement is repeated many times until the system reaches an equilibrium state. More details about the SR method can be found in $[16,21]$.

Initial configuration is generated by randomly placing all atoms in a simulation box. Then, the sample is relaxed over several thousand steps until the system attains the equilibrium, for example, the energy of system fluctuates around a constant value and the pressure is equal to zero.The model A is constructed by relaxing with $d r=0.4 \AA$ over 200 SR steps and then is treated with $d r=0.01 \AA$ by $10^{6}$ SR steps. To investigate the relaxation effect, two additional samples (samples B and C) are prepared with the same density as sample A, but their potential energy is lower (more stable state). Note that the lower the energy of the system the bigger its relaxation degree. More stable samples B and C can be constructed many times with relaxing the model A that has $d r=0.4 \AA$, which likes the shaking of atomic structure. Then, they are again relaxed with $d r=0.01 \AA$ until the system reaches a new equilibrium.

Four neighboring atoms form a tetrahedron and have a circumsphere (CSP) whose surface passes through the vertices of this tetrahedron. Consider only such tetrahedron with CSP that does not contain any atom inside. For example, those atoms are the nearest neighboring. Let $R_{\text {CSP }}$ and $n$ be the radius of CSP and the number of atoms on the surface of CSP, respectively. The atom on surface of CSP is determined as one that is located from the center of CSP at a distance in range of $R_{\mathrm{CSP}} \pm 0.1 \AA$. hereafter we call it $n$-simplex (see Figures 1(a) and 1(b)). If $n$ and $R_{\text {CSP }}$ is large enough, then $n$ atoms form an atomic cage like a bubble (BB), for example, a large group of atoms gathered around a large void. The $\mathrm{BB}$ is unstable and it may break up leading to diffusion. Therefore, it is interesting to clarify which one among $n$-simplexes is BB and how BB breaks up. Hence, the next calculation is performed as follows: first for every simplex, we test all $n$ atoms to perform jump inside the CSP. Second, the atom selected to move is inserted inside CSP and the system is relaxed until it reaches equilibrium.

\section{Results and Discussion}

3.1. The Sample. Figure 2 shows the radial distribution function (RDF) $g(r)$ of the amorphous samples. As shown in Figure 2, although the energy per atom of the considered samples changes from -0.9336 to $-0.9534 \mathrm{eV}$, the RDF $g(r)$ for the considered samples is identical. This indicates the nonsensitivity of the function $g(r)$ to the change in local microstructure of AMs. This result further indicates that the relaxation degree almost does not affect RDF, but it is reflected by the concentration of simplex. To test the validity of the constructed samples, we have compared our obtained $\mathrm{RDF}$ with the experimental data. As shown from Figure 3 the structural characteristics of our samples are in accordance with the experiment data [22]. In addition, the function $g(r)$ has a splitting second peak, which is often thought to be related to the icosahedrons in amorphous systems $[17,18,20]$. The main characteristics of the samples are listed in Table 1. The comparison with experiment shows agreement in the position and height of the first peak of RDF, which indicates the reality of the constructed sample. 


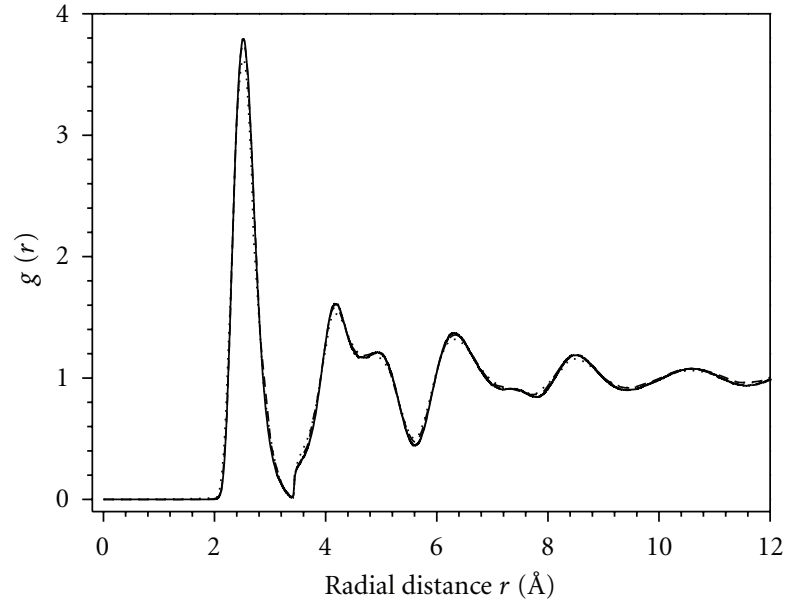

... Sample A

- - - Sample B

- Sample C

FIGURE 2: The radial distribution function of the obtained samples.

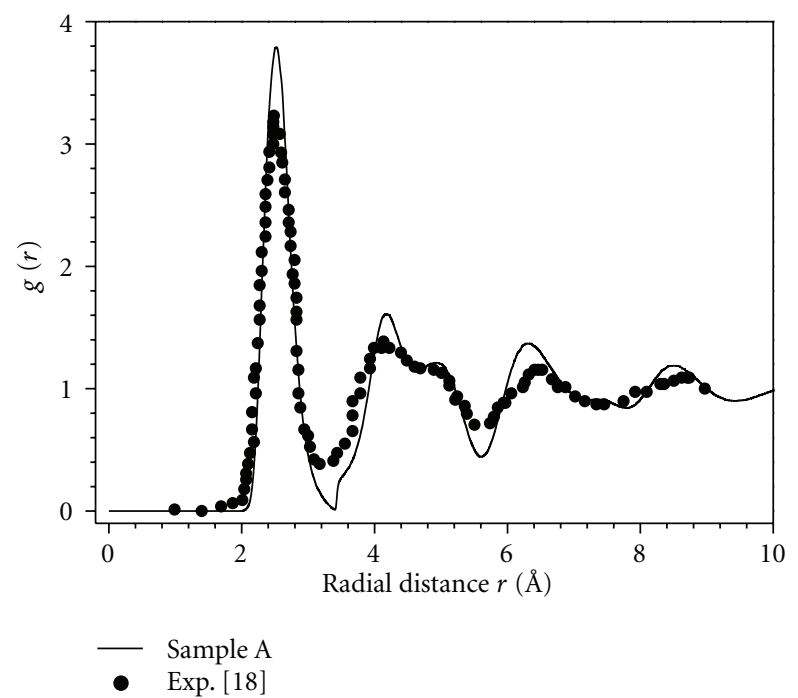

FIgure 3: The radial distribution function of sample A and the experimental data in [18].

TABle 1: Structure characteristics of obtained samples. Here, $r_{i}$ is the position of $i$ th peak and $g(r)$ is the height of first peak of the RDF.

\begin{tabular}{lcccccc}
\hline Systems & $r_{1}$ & $r_{2} / r_{1}$ & $r_{3} / r_{1}$ & $r_{4} / r_{1}$ & $r_{5} / r_{1}$ & $g(r)$ \\
\hline Sample A & 2.51 & 1.67 & 1.99 & 2.52 & 3.39 & 3.64 \\
Sample B & 2.52 & 1.67 & 1.99 & 2.51 & 3.38 & 3.75 \\
Sample C & 2.50 & 1.66 & 2.01 & 2.54 & 3.44 & 3.78 \\
${ }^{\mathrm{a} C o}$ & 2.49 & 1.69 & 1.93 & 2.49 & 3.35 & 3.20 \\
bCo & - & 1.65 & 1.90 & 2.57 & 3.46 & - \\
\hline
\end{tabular}

a,b Experimental data in [22-24].

3.2. Simplex and Role of Simplex. Table 2 presents the energy per atom and fraction of simplex found in the obtained samples. One can see that the energy per atom decreases
TABLe 2: The energy per atom $(\varepsilon)$ and the fraction of $n$-simplexes in the obtained samples. Here, $n$ is number of atom in one simplex.

\begin{tabular}{|c|c|c|c|c|}
\hline Systems & $4\left(\times 10^{0}\right)$ & $5\left(\times 10^{-1}\right)$ & $6\left(\times 10^{-2}\right)$ & $\geq 7\left(\times 10^{-3}\right)$ \\
\hline Sample A -0.9336 & 0.961 & 0.374 & 0.173 & 0.02 \\
\hline Sample B -0.9462 & 0.963 & 0.358 & 0.167 & 0.00 \\
\hline Sample C -0.9534 & 0.964 & 0.339 & 0.164 & 0.00 \\
\hline
\end{tabular}

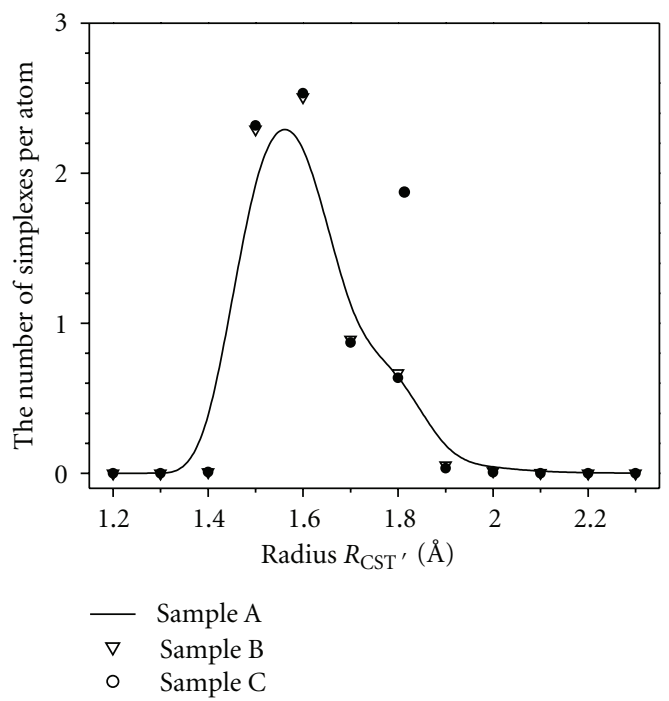

FIGURE 4: The number of simplexes per atom as function of radius $R_{\text {CST }}$.

from -0.9336 to $-0.9534 \mathrm{eV}$, upon relaxation degree. The fraction of 4-simplex increases as the sample potential energy decreases, that is, the more stable state is, bigger the number of 4-simplexes is. For other kinds of simplex, we observe the opposite trend. Thus, it means that due to fast quenching from liquid the parking of atoms in AM is not efficient. Therefore, there is always an amount of structural defects like large void (free volume). The relaxation is accompanied by annihilation of those defects. As mentioned above, the bigger number $n$ is, the bigger the size of simplex and void inside it is. Therefore, Table 2 specifies that the monotony decrease in the number of $n$-simplex with $n>4$ from less relaxed (sample A) to well relaxed state (sample C) indicates the annihilation of structural defects in amorphous matrix. This result also can be seen in the radius distribution of simplex as in Figure 4. From Figure 4, one can see clearly the significant decrease in the number of large simplex $\left(R_{\mathrm{CSP}}>1.8 \AA\right)$ in the sample A as compared to the sample $\mathrm{C}$. We proposed that the large simplex $(n=6,7,8)$ is broken up into 4 -simplex under the relaxation degree. So as the concentration of 4-simplex increases, the large simplex decreases.

We now turn to discuss the role of large simplex for diffusivity in AM. For each simplex, we examine the potential energy profile (PEP) for neighboring atoms moving into CSP of the simplex. The PEP is determined as follows: each of $n$ atoms of the simplex is taken and then we move this atom step by step towards the center of CSP. The step length is set to be $0.02 \AA$. At each step, the potential 


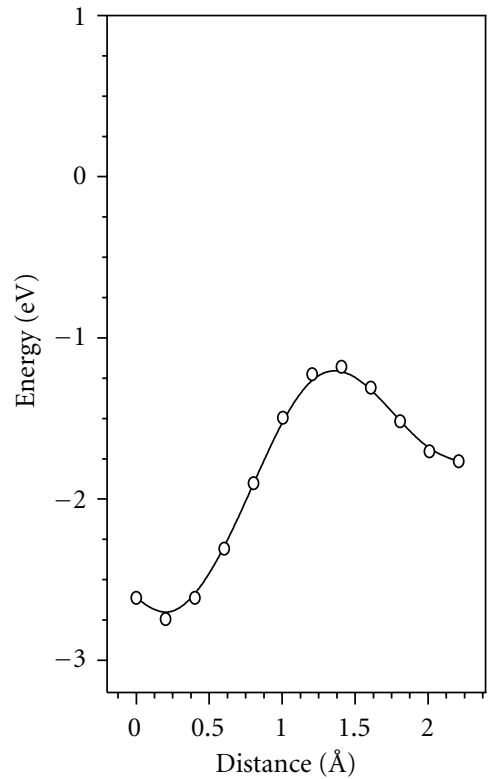

(a)

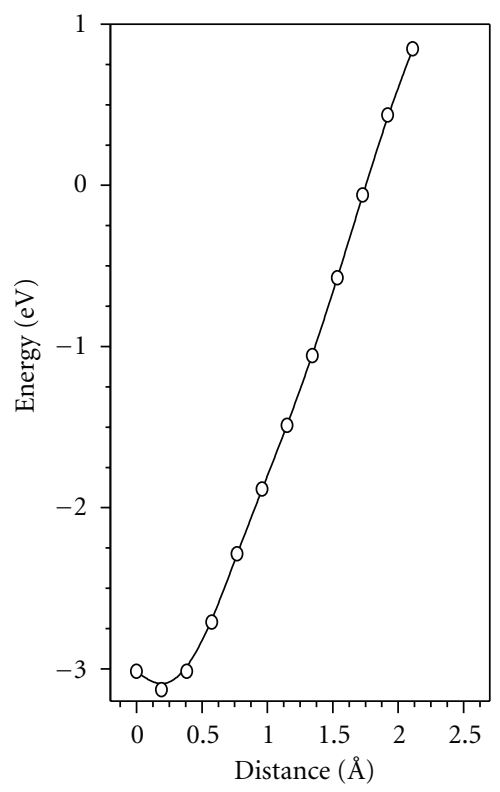

(b)

FIgure 5: Potential energy profiles for atom moving into simplex.

energy of the moving atom (MA) is recorded. Finally, we obtain the PEP for MA. Figure 5 displays the typical PEPs for several simplexes detected in our samples. Curve $(b)$ (Figure 5(b)) shows the monotonous increase in the MA energy indicating that the MA could not jump into the CSP due to very high potential barrier. Curve (a) (Figure 5(a)), in converse, have a pronounced maximum. Here, the barrier height is determined by the maximum and initial points in PEP. They are like the PEP for tracer atom jumping in vacancy incrystalline lattice. Therefore, the MA can jump into CSP, and the simplex plays a role of the diffusion vehicle assisting tracer atom to move in amorphous matrix. The
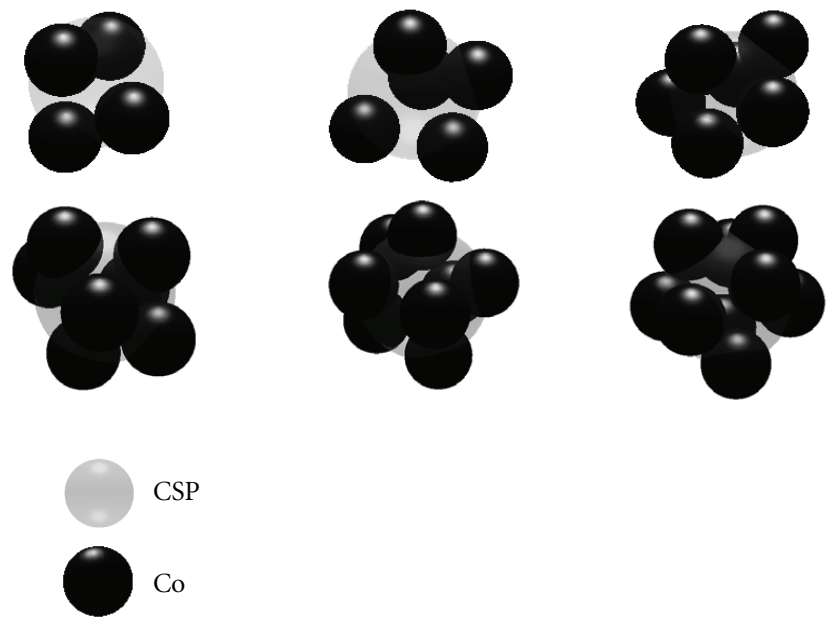

FIGURE 6: The snapshot of typical bubbles detected in the obtained sample.

simplex having PEP like curves $a$, with corresponding MA, is called the bubbles (BB) and the diffusing atom (DA), respectively. Figure 6 shows some typical $n$-bubbles in the obtained samples. The number of $\mathrm{BB}\left(n_{\mathrm{BB}}\right)$ is found in Table 3. As shown in Table 3, the number of BB decreases depending upon relaxation degree. The dependence of the number of DA on their corresponding barrier height is shown in Figure 7. Most frequent barrier height lies in the interval of $0.5-1.9 \mathrm{eV}$. Because the diffusion coefficient in general is proportional to $n_{\mathrm{BB}}$, it is given as follows:

$$
D=\frac{1}{6} f v_{0}\left\langle d^{2}\right\rangle \exp \left(\frac{\Delta s_{m}}{k_{B}}\right) n_{\mathrm{BB}} \exp \left(-\frac{E_{m}}{k_{B} T}\right) .
$$

Here, $f$ is the correlation factor, $v_{0}$ is the attempt frequency, $d$ is the jump length, $\Delta s_{m}, E_{m}$ are the effective migration entropy and energy for diffusion in disordered media, respectively, $k_{B}$ is the Boltzmann constant. Note that the parameter $\left\langle d^{2}\right\rangle$ represents the mean square displacement of different jump lengths. Because the SR model is in fact the molecular dynamic (MD) model in the limit of zero temperature, the number of simplexes found in the samples is independent of temperature and it varies only with the relaxation degree. As a result, the activation energy is the migration energy $\left(E_{m}\right)$, and the preexponential factor $D_{0}$ is given as

$$
D_{0}=\frac{1}{6} f v_{0}\left\langle d^{2}\right\rangle \exp \left(\frac{\Delta s_{m}}{k_{B}}\right) n_{B B}
$$

Furthermore, the decrease in diffusion coefficient under relaxation is ascribed by the annihilation of the bubbles. Therefore, the decrease in Co diffusivity is found to be 27 times for samples A and C.

Several experimental findings shows that (i) sudden temperature change during diffusion annealing results in instantaneous change of diffusion coefficient $\left(D_{R}\right)$ and (ii) The self-diffusion enthalpy $H$ of transition metal for certain amorphous alloy seems to be the migration enthalpy, but 
TABLE 3: The number of $\mathrm{BB}\left(n_{\mathrm{BB}}\right)$; the pre-exponential factor $\left(D_{0}\right)$ at $560 \mathrm{~K}$ in obtained samples.

\begin{tabular}{lcc}
\hline Systems & $n_{\mathrm{BB}}\left(\times 10^{-3}\right)$ & $D_{0}\left(\mathrm{~m}^{2} / \mathrm{s}\right)$ \\
\hline Samples A & 8.39 & $9.3 \times 10^{-6}$ \\
Samples B & 1.34 & $6.1 \times 10^{-7}$ \\
Samples C & 0.31 & $1.1 \times 10^{-7}$ \\
${ }^{\mathrm{c}} \mathrm{Co}_{89} \mathrm{Zr}_{11}$ & - & $8.0 \times 10^{-7}$ \\
${ }^{\mathrm{d}} \mathrm{Co}_{79} \mathrm{Nb}_{14} \mathrm{~B}_{7}$ & - & $3.3 \times 10^{-6}$ \\
\hline
\end{tabular}

c,d Experimental data in $[25,26]$.

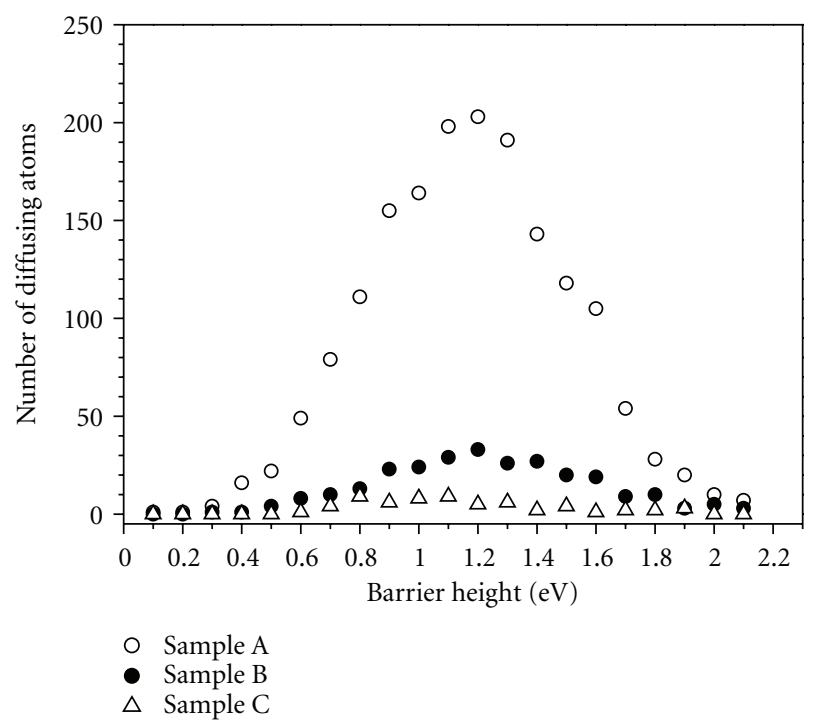

FIgURE 7: The distribution of barrier height of diffusing atom.

not a sum of migration and formation enthalpy of diffusion vehicles as it is the case of self-diffusion in crystal [3]. These experimental observations are interpreted as a result of direct diffusion mechanism occurring in relaxed amorphous sample. Our simulation result shows possible diffusivity via the bubble. It is consistent with the experiment because the concentration of bubbles is independent of temperature, and they vary only with relaxation degree. So, the activation energy for diffusion via the bubble is equal to the migration energy $\left(E_{m}\right)$.

To estimate the parameter $\left\langle d^{2}\right\rangle$ in (2), we replace DA into CSP and then relax the system until a new equilibrium is attained. This procedure for convenience is called the remove diffusion atom (RDA). The distribution of mean square displacements for 100 RDAs is displayed in Figure 8. For sample $C$, the parameter $\left\langle d^{2}\right\rangle$ of most RDAs is less than $10 \AA^{2}$ (92 atoms). Because the jump length of DA lies in the interval of 2.0-3.0 $\AA$, the contribution of DA to $\left\langle d^{2}\right\rangle$ will be essential and the RDA locates only in the small region nearby the bubble. In the case of sample A, we observe a very large value of $\left\langle d^{2}\right\rangle$ (more than $10 \AA^{2}$ ), which represents a collective movement of a large number of atoms. Obviously, the atomic movement is spread over a large volume inside the

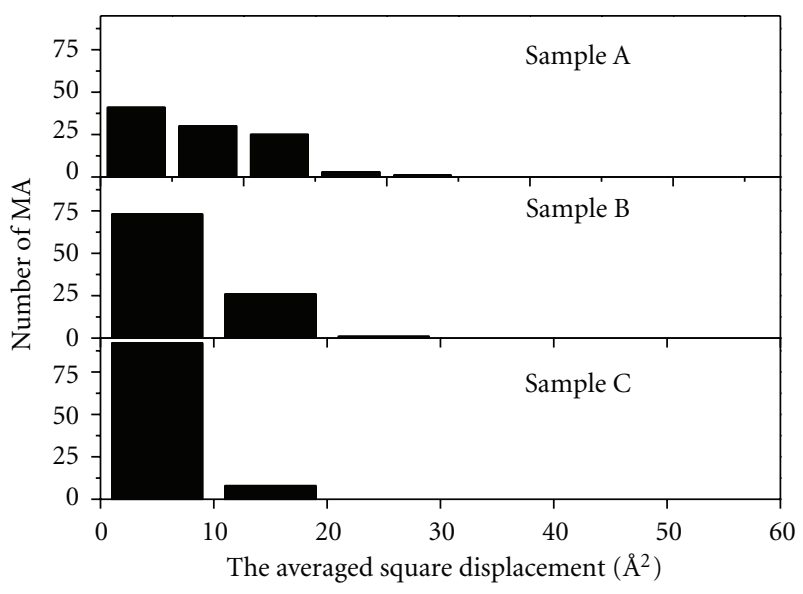

Figure 8: The distribution of averaged square displacement of moving atoms.

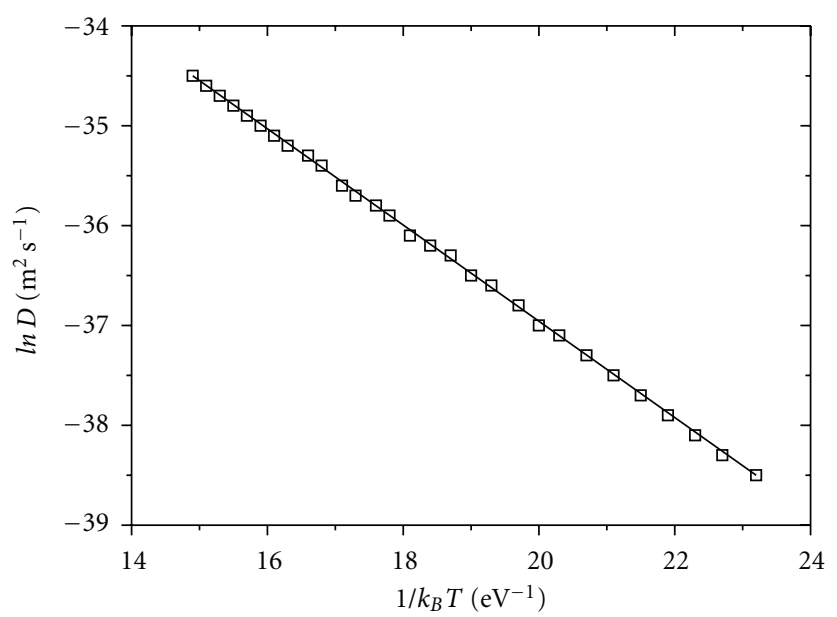

Calculated by (2)

Figure 9: The temperature dependence of diffusion coefficient; a line is drawn to show the Arrhenius behavior.

amorphous matrix as shown in Figure 1(d). Therefore, one may see two distinct diffusion mechanisms occurring in the as-quenched (sample A) and well-relaxed metastable states (sample C). The first one is like the hoping mechanism via bubble. The second one relates to the collective mechanism involving a large number of atoms in each elemental diffusion movement. Assume that $f=1, \exp \left(\Delta \mathrm{s}_{\mathrm{m}} / k_{B}\right) \approx$ $1, v_{0}=10^{12} s^{-1},\left\langle d^{2}\right\rangle \approx 10 \AA^{2}$ for sample $\mathrm{C}$ and $100 \AA^{2}$ for sample A. The diffusion coefficient of Co atom can be estimated on the base of (2). Although the barrier heights for different jumps vary from 0.5 to $1.9 \mathrm{eV}$, the $D_{\mathrm{Co}}$ diffusion coefficient is found to obey the Arrehenius behavior as shown in Figure 9. According to (3), the pre-exponential factor is calculated as shown in Table 3 . The calculation data is consistent with the experimental data for the amorphous samples $[25,26]$. 


\section{Conclusions}

A comprehensive study on both the number of simplexes and a bubbles diffusion mechanism in AM has been carried out using SR simulation with the pair Pak-Doyama inter-atomic potential upon the change of relaxation degree. Conclusions are as followed:

(i) The microstructure of the cobalt samples containing $2 \times 10^{5}$ atoms is in agreement with the experimental data. The fraction of 4-simplex increases and $n$ simplex $(n>4)$ decreases depending upon relaxation degree. The simulation reveals that a large number of bubbles vary with relaxation degree in AM.

(ii) A bubbles diffusion mechanism in AM is proposed for the elemental atomic movement, which includes a jump of neighboring atom into the bubbles and then collective displacement of a number of atoms. It may be seen two distinct diffusion mechanisms occurring in the as-quenched and well-relaxed samples. The decrease in pre-exponential factor upon relaxation is ascribed to the partial annihilation of bubbles. The obtained pre-exponential factor of Co atom is found to be in accordance with the experiment data.

\section{References}

[1] J. Horváth, J. Ott, K. Pfahler, and W. Ulfert, "Tracer diffusion in amorphous alloys," Materials Science and Engineering, vol. 97, pp. 409-413, 1988.

[2] J. Pavlovsky, W. Ulfert, and W. Frank, "Self-diffusion of ${ }^{58} \mathrm{Co}$ in amorphous $\mathrm{Co}_{79} \mathrm{Nb}_{14} \mathrm{~B}_{7}$ during isothermal crystallization," Materials Chemistry and Physics, vol. 36, no. 3-4, pp. 383-388, 1994.

[3] W. Frank, A. Horner, P. Scharwaechter, and H. Kronmiiler, "Diffusion mechanisms in amorphous alloys," Materials Science and Engineering, vol. 97, pp. 415-418, 1988.

[4] A. K. Tyagi, M. P. Macht, and V. Naundorf, "Diffusion coefficients of ${ }^{63} \mathrm{Ni}$ in $\mathrm{Fe}_{40} \mathrm{Ni}_{40} \mathrm{~B}_{20}$ metallic glass," Acta Metallurgica et Materialia, vol. 39, no. 4, pp. 609-617, 1991.

[5] T. Schuler, J. Pavlovskýa, P. Scharwaechter, W. Ulfert, and W. Frank, "Change of the self-diffusion of ${ }^{58} \mathrm{Co}$ in $\mathrm{Co}_{79} \mathrm{Nb}_{14} \mathrm{~B}_{7}$ during transition from the amorphous to the nanocrystalline phase," Nanostructured Materials, vol. 6, no. 5-8, pp. 863-867, 1995.

[6] S. Flege, U. Fecher, and H. Hahn, "Diffusion in amorphous NiZrAl alloys," Journal of Non-Crystalline Solids, vol. 270, no. 1-3, pp. 123-128, 2000.

[7] A. Grieschea, T. Zumkleya, M.-P. Machta, S. Suzukib, and G. Frohbergb, "Diffusion in PdCuNiP alloys from the amorphous to the liquid state," Materials Science and Engineering A, vol. 375-377, pp. 285-287, 2004.

[8] S. Chakravarty, M. Gupta, A. Gupta et al., "Fe and N selfdiffusion in amorphous FeN: a SIMS and neutron reflectivity study," Acta Materialia, vol. 57, no. 4, pp. 1263-1271, 2009.

[9] S. K. Sharma, S. Banerjee, Kuldeep, and A. K. Jain, "Diffusion measurements in the $\mathrm{Fe}_{82} \mathrm{~B}_{18}$ amorphous alloy by rutherford backscattering spectrometry," Acta Metallurgica, vol. 36, no. 7, pp. 1683-1690, 1988.

[10] W. D. Roos, J. D. Plessis, and G. N. van Wyk, "Diffusion of silicon in Fe-based amorphous and crystalline alloys," Applied Surface Science, vol. 40, no. 4, pp. 303-307, 1990.
[11] Y. Limoge, "Role of energetic disorder on diffusion in amorphous alloys," Journal of Non-Crystalline Solids, vol. 117-118, no. 2, pp. 605-608, 1990.

[12] G. Ruitenberg, P. de Hey, F. Sommerb, and J. Sietsma, "Pressure dependence of the free volume in amorphous $\mathrm{Pd}_{40} \mathrm{Ni}_{40} \mathrm{P}_{20}$ and its implications for the diffusion process," Materials Science and Engineering A, vol. 226-228, pp. 397-400, 1997.

[13] Y. Limoge, "Activation volume for diffusion in a metallic glass," Acta Metallurgica et Materialia, vol. 38, no. 9, pp. 1733-1742, 1990.

[14] R. S. Averback, "Defects and diffusion in amorphous alloys," MRS Bulletin, vol. 16, pp. 47-52, 1991.

[15] Y. Limoge, "Microscopic and macroscopic properties of diffusion in metallic glasses," Materials Science and Engineering A, vol. 226-228, pp. 228-236, 1997.

[16] P. K. Hung, H. V. Hue, and L. T. Vinh, "Simulation study of pores and pore clusters in amorphous alloys $\mathrm{Co}_{100-x} \mathrm{~B}_{x}$ and $\mathrm{Fe}_{100-y} \mathrm{P}_{y}$," Journal of Non-Crystalline Solids, vol. 352, no. 3031, pp. 3332-3338, 2006.

[17] A. Zhu, G. J. Shiflet, and S. J. Poon, "Diffusion in metallic glasses: analysis from the atomic bond defect perspective," Acta Materialia, vol. 56, no. 14, pp. 3550-3557, 2008.

[18] P. K. Leung and J. G. Wright, "Structural investigations of amorphous transition element films: II. Chromium, iron, manganese and nickel," Philosophical Magazine, vol. 30, no. 5, pp. 995-1008, 1974.

[19] V. Naundorf, M. P. Macht, A. S. Bakai, and N. Lazarev, "The pre-factor, $\mathrm{D}_{0}$, of the diffusion coefficient in amorphous alloys and in grain boundaries," Journal of Non-Crystalline Solids, vol. 224, no. 2, pp. 122-134, 1998.

[20] P. K. Hung, P. H. Kien, and L. T. Vinh, "Evidence of 'microscopic bubbles' and a new diffusion mechanism for amorphous alloys," Journal of Physics, vol. 22, Article ID 035401, pp. 1-5, 2010.

[21] J. Sietsma and B. J. Thijsse, "Characterization of free volume in atomic models of metallic glasses," Physical Review B, vol. 52, no. 5, pp. 3248-3255, 1995.

[22] Y. Waseda and S. Tamaki, "Structures of 3D-transition metals in the liquid state," Philosophical Magazine, vol. 32, no. 2, pp. 273-281, 1975.

[23] P. K. Leung and J. G. Wright, "Structural investigations of amorphous transition element films I. Scanning electron diffraction study of cobalt," Philosophical Magazine, vol. 30, no. 1, pp. 185-194, 1974.

[24] P. K. Leung and J. G. Wright, "Structural investigations of amorphous transition element films-2. Chromium, iron, manganese and nickel," Philosophical Magazine, vol. 30, no. 5, pp. 995-1108, 1974.

[25] V. Naundorf, M.-P. Machta, A. S. Bakaib, and N. Lazarevb, "The pre-factor, $\mathrm{D}_{0}$, of the diffusion coefficient in amorphous alloys and in grain boundaries," Journal of Non-Crystalline Solids, vol. 224, no. 2, pp. 122-134, 1998.

[26] J. Pavlovsky and J. Cermak, "Diffusion of ${ }^{59} \mathrm{Fe}$ in amorphous $\mathrm{Co}_{79} \mathrm{Nb}_{14} \mathrm{~B}_{7}$ alloy," Scripta Metallurgica et Materialia, vol. 30, no. 10, pp. 1317-1320, 1994. 

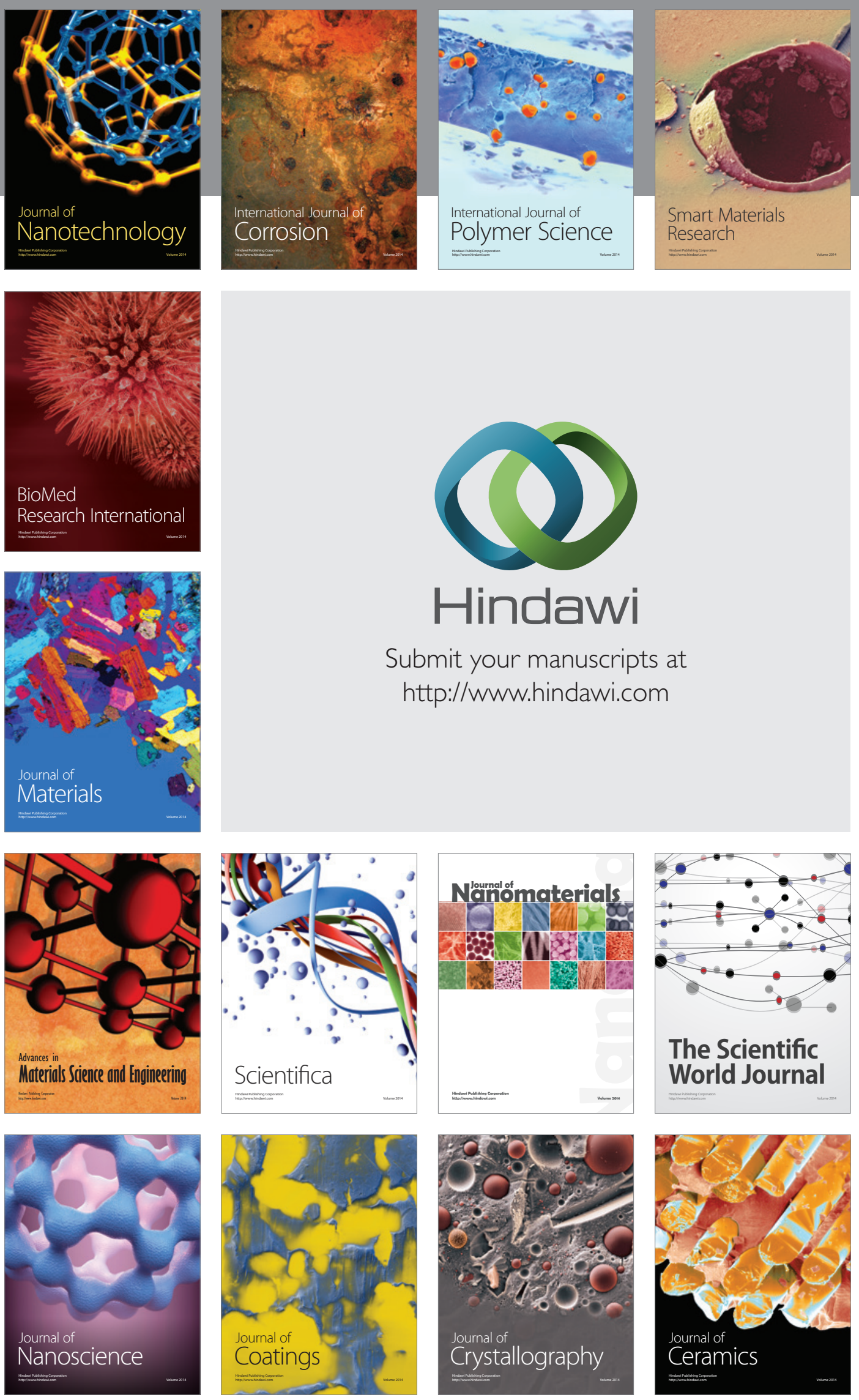

The Scientific World Journal

Submit your manuscripts at

http://www.hindawi.com

\section{World Journal}

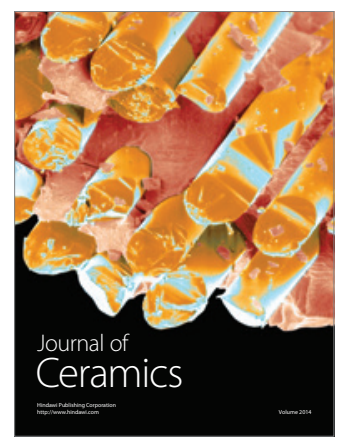

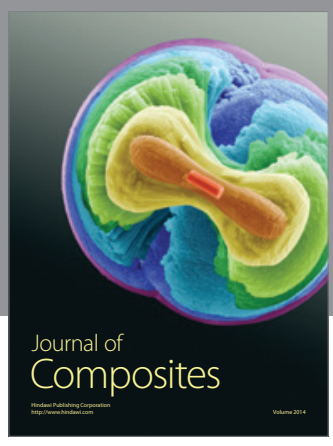
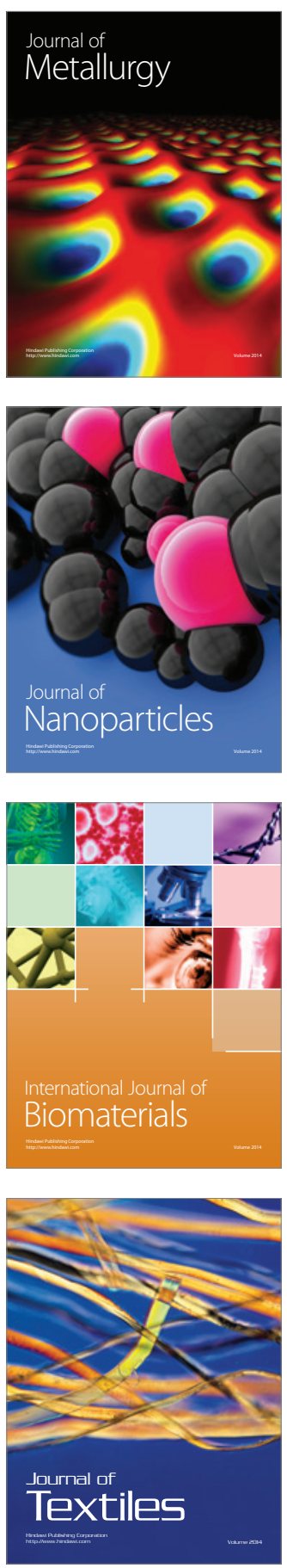